\title{
Solitary fibrous tumor of the salivary gland: A case report
}

\author{
YINGWEN ZHOU, JIN ZHENG, QINGQIANG ZHU, WEI XIA and SANTOSH KUMAR BHAGAT \\ Department of Medical Imaging, Subei People's Hospital, Medical School of Yangzhou University, \\ Yangzhou, Jiangsu 225001, P.R. China
}

Received January 16, 2015; Accepted October 6, 2015

DOI: $10.3892 / \mathrm{ol} .2015 .3948$

\begin{abstract}
Solitary fibrous tumors (SFTs) are rare soft-tissue tumors of mesenchymal origin. Occasionally, these lesions have been indicated to associate with the salivary glands. Through the analysis of magnetic resonance imaging sequences, the present study reports a case of a solitary salivary gland lesion, demonstrating a well-circumscribed, soft-tissue tumor with marked signal changes and homogenous enhancement. SFT should be considered as a differential diagnosis when a solid mass exhibiting hypointensity on T1-weighted images and hyperintensity on T2-weighted images has been detected in the salivary gland. Due to the potentially malignant nature of SFTs, it is necessary for radiologists to improve their understanding of such lesions.
\end{abstract}

\section{Introduction}

A solitary fibrous tumor (SFT) is a rare form of tumor that typically develops in the pleura. SFTs that establish in the salivary gland are particularly rare, with imaging studies describing only a few known cases $(1,2)$. Due to the rarity of the tumor, the incidence of such in the salivary gland remains unclear. The association between pathological morphology and clinical symptoms is non-specific. SFTs are unpredictable and the diagnosis remains challenging. Additionally, $10-15 \%$ of tumors advance to become malignant or metastasize, and can recur (3). SFTs in the head and neck region are also uncommon, with the characteristics of tumors in this location not clearly understood (4). Magnetic resonance imaging (MRI) features are also relatively non-specific. Wignall et al (5) described that $89 \%$ of tumors presented with high signal intensities on $\mathrm{T} 2$-weighted images and

Correspondence to: Professor Jin Zheng, Department of Medical Imaging, Subei People's Hospital, Medical School of Yangzhou University, 98 West Nantong Road, Yangzhou, Jiangsu 225001, P.R. China

E-mail: zhj65220@163.com

Key words: solitary fibrous tumor, salivary gland, magnetic resonance imaging, immunohistochemistry intermediate signal intensities on T1-weighted images in a review of previous studies. Areas of low signal intensity were observed in larger tumors, resulting from flow voids in prominent perilesional vessels. Collagen fibroblasts and tumor degeneration have been suggested to be accountable for such variable signal intensities (6). Previous studies have reported linear or curvilinear hypointense areas within SFTs on T2-weighted images, and such lesion morphology has been demonstrated to correlate with hypocellular densities near the collagenous sclerotic area $(6,7)$.

Although further investigation is required on the incidence of SFTs in this location, the present study describes a lesion in the right salivary gland and the subsequent MRI findings.

\section{Case report}

In October 2013, a 48-year-old woman was referred to the Subei People's Hospital (Yangzhou, China), exhibiting a large, soft salivary mass that had been identified 20 days earlier. The physical examination was unremarkable, with the exception of the palpable mass in the right jaw. The tumor extended from under the jaw to the right side of the bottom of the mouth, and its quality reflected a well-defined, poorly-mobilized lesion with no facial nerve symptoms. The patient maintained a bilateral symmetrical face with normal mouth opening and occlusion.

A plain MRI scan confirmed the presence of a well-circumscribed, irregular, soft-tissue mass measuring $71 \times 29 \mathrm{~mm}$, located in the right salivary area, associated with the right salivary gland (Fig. 1). The tumor exhibited marked and homogeneous enhancement following intravenous injection of contrast material. The adjacent bone was not involved and the lymph nodes in the carotid sheath area were observed. A benign tumor was suspected and subsequently removed by local excision. The surgical specimen was examined and an irregular-shaped mass with a complete capsule was detected. Microscopic examination demonstrated spindle cells and a collagen component in the mesenchyme, with reduced cytoplasm (Fig. 2A and B). Immunohistochemical analysis indicated neoplastic cells that were strongly positive for cluster of differentiation (CD)34 monoclonal antibody (Fig. 2C and D). The cytoplasm (brown) was identified as strongly positive for vimentin monoclonal antibody. Thus, the case was diagnosed as an SFT. Written informed consent was obtained from the patient for publication of this study. 

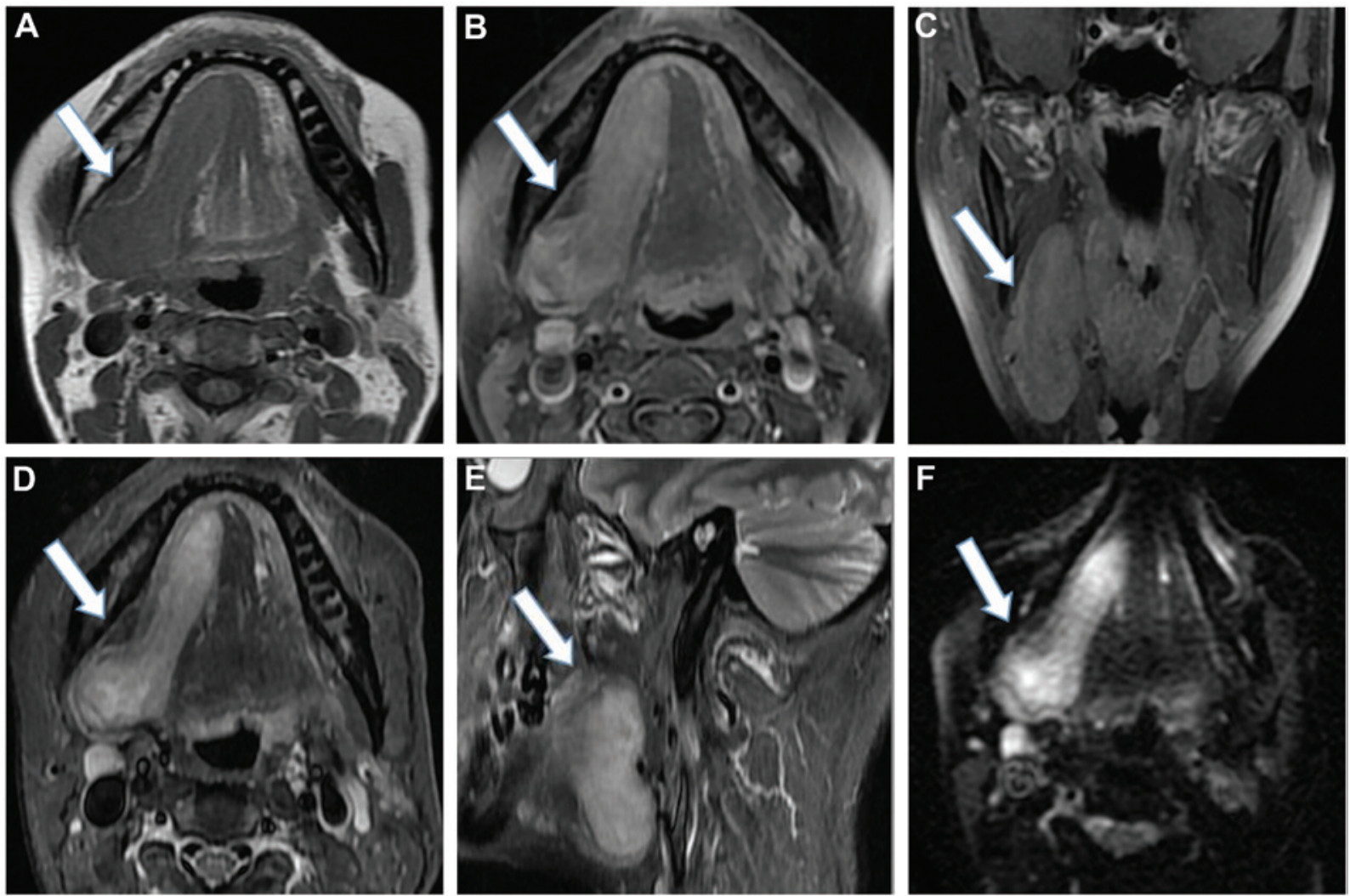

Figure 1. MRI of the tumor measuring 71x29 $\mathrm{mm}$ in the right salivary area. (A) Axial T1-weighted MRI demonstrating a well-defined mass invading the adjacent salivary gland, which is hypointense compared with the adjacent muscle. (B) Contrast-enhanced axial image scan illustrating homogeneous enhancement of the mass (arrow). (C) Contrast-enhanced coronal image scan demonstrating homogeneous enhancement of the mass. (D) Axial fat-suppressed T2-weighted MRI demonstrating a predominantly hyperintense mass. (E) Sagittal fat-suppressed T2-weighted MRI exhibiting isointense to hyperintense signal intensities in the mass. (F) Diffusion-weighted imaging illustrating the hyperintensified lesion. Arrows indicate the tumor mass. MRI, magnetic resonance imaging.

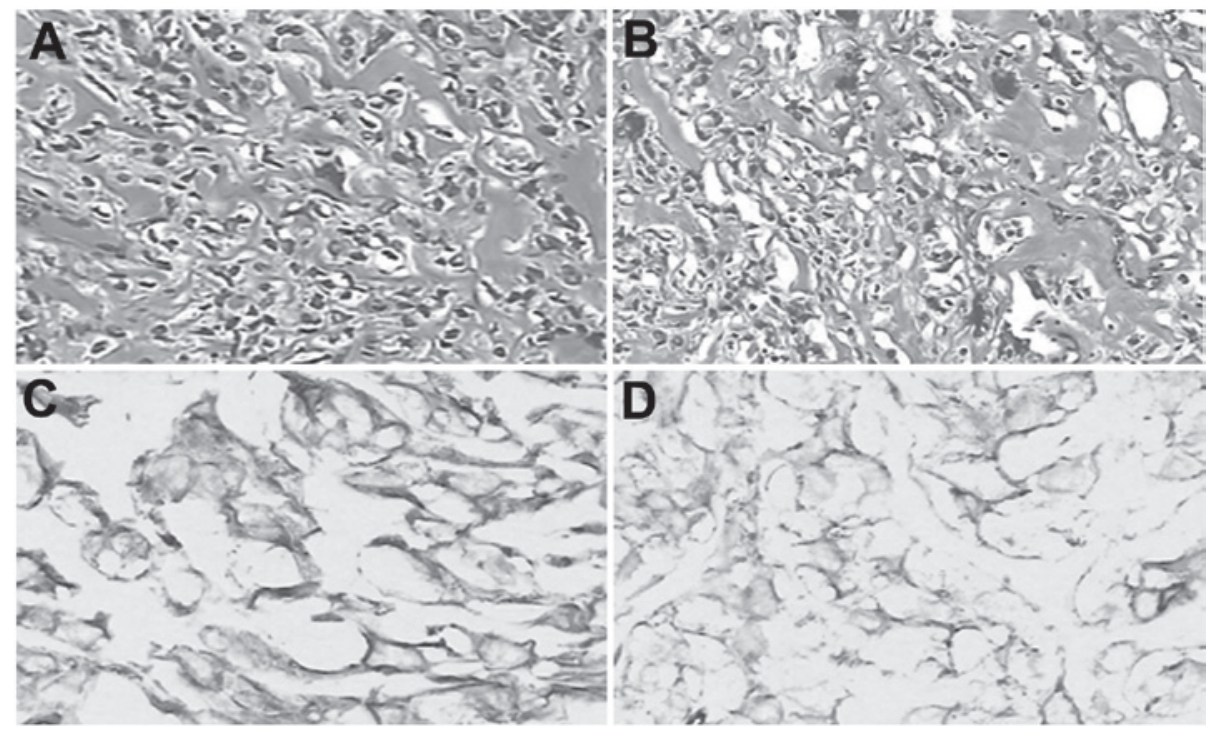

Figure 2. (A and B) Photomicrograph demonstrating a solitary fibrous tumor composed of spindle cells (hematoxylin and eosin staining). (C and D) Immunohistochemical staining of neoplastic cells positive for cluster of differentiation 34 monoclonal antibody in the membrane and cytoplasm (brown), and cells positive for vimentin monoclonal antibody in the cytoplasm (brown). Magnification, $\mathrm{x} 400$.

\section{Discussion}

SFTs primarily occur in adults ranging from 30 to 64 years old (6). SFTs are rare lesions that usually occur in the pleura and were first described by Klemperer and Rabin in 1931 (8).
Previous studies have also reported such lesions in the head and neck regions, and $\sim 80$ cases have been described regarding SFTs located in the oral cavity (9). The risks of local recurrence and metastasis are associated with tumor size and histological grade of surgical resection margins $(3,5,10)$. 
In general, 79-100\% positivity for CD34 on immunohistochemistry is considered a highly specific marker of SFTs (11). In addition, malignant SFTs tend to exhibit decreased CD34 immunoreactivity and overexpress p53, S-100, keratin and vimentin (12-14). However, separating SFTs arising in the head and neck region from those arising at other sites by histopathological or immunophenotypical features is not straightforward (15).

In the present case, the salivary gland SFT presented as a well-circumscribed, submucosal mass that was asymptomatic and slow-growing, with normal staining. SFTs can often be confused with other salivary gland conditions, including submandibular gland inflammation and sublingual gland cysts (1).

In the head and neck regions, the radiological findings of SFTs are not specific. However, a number of SFTs are detected incidentally through radiological examinations, or when the symptoms appear associated with a mass in the surrounding tissues (6). The components of the tumor itself may cause the variable intensity observed on MRI images (6). However, radiological differentiation of SFTs from other salivary gland tumors, including pleomorphic adenoma, is difficult. Therefore, in clinical practice, imaging techniques combined with immunohistochemical and pathological results may be advantageous for diagnosis.

SFTs are often observed as a soft-tissue attenuation on imaging, comprised of a well-marginated, lobulated mass when the tumor has prominent collateral feeding vessels or a visible fatty component. Such features alert the radiologist to a possible SFT diagnosis. When the tumor is $\geq 10 \mathrm{~cm}$ in size with central necrosis and effusion, it is likely to be a malignant tumor $(5,16,17)$.

The most important prognostic factor in treating SFTs is treatment with a complete resection $(18,19)$. Due to the high probability of late recurrence, long-term follow-ups are required (18).

In conclusion, the present study findings suggest that an SFT should be considered in the differential diagnosis of a well-marginated lesion with salivary gland association, a soft-tissue component, isointensity to hyperintensity on T2-weighted images and a high signal intensity on diffusion-weighted images, including homogeneous contrast-enhancement.

\section{References}

1. Ferreiro JA and Nascimento AG: Solitary fibrous tumor of the major salivary glands. Histopathology 28: 261-264, 1996.

2. ChoKJ,RoJY,ChoiJ,ChoiSH,Nam SY andKimSY:Mesenchymal neoplasms of the major salivary glands: Clinicopathologcal features of 18 cases. Eur Arch Otorhinolaryngol 265 (Suppl 1): S47-S56, 2008.
3. Garcia-Bennett J, Olivé CS, Rivas A, Domínguez-Oronoz R and Huguet P: Soft tissue solitary fibrous tumor. Imaging findings in a series of nine cases. Skeletal Radiol 41: 1427-1433, 2012.

4. Yamada H, Hamada Y, Fujihara H, Fukami K, Mishima K, Nakaoka K, Kumagai K and Imamura E: Solitary fibrous tumor of the buccal space resected in combination with coronoidectomy. Oral Surg Oral Med Oral Pathol Oral Radiol 114: e9-e14, 2012.

5. Wignall OJ, Moskovic EC, Thway $\mathrm{K}$ and Thomas JM: Solitary fibrous tumors of the soft tissues: Review of the imaging and clinical features with histopathologic correlation. AJR Am J Roentgenol 195: W55-W62, 2010.

6. Kim HJ, Lee HK, Seo JJ, Kim HJ, Shin JH, Jeong AK, Lee JH and Cho KJ: MR imaging of solitary fibrous tumors in the head and neck. Korean J Radiol 6: 136-142, 2005.

7. Jeong AK, Lee HK, Kim SY and Cho KJ: Solitary fibrous tumor of the parapharyngeal space: MR imaging findings. AJNR Am J Neuroradiol 23: 473-475, 2002.

8. Klemperer P and Rabin CB: Primary neoplasm of the pleura: A report of 5 cases. Arch Pathol (Chic) 1: 11-28, 1931.

9. Amico P, Colella G, Rossiello R, Maria Vecchio G, Leocata P and Magro G: Solitary fibrous tumor of the oral cavity with a predominant leiomyomatous-like pattern: A potential diagnostic pitfall. Pathol Res Pract 206: 499-503, 2010.

10. Daigeler A, Lehnhardt M, Langer S, Steinstraesser L, Steinau HU, Mentzel T and Kuhnen C: Clinicopathological findings in a case series of extrathoracic solitary fibrous tumors of soft tissues. BMC Surg 6: 10, 2006.

11. Ali SZ, Hoon V, Hoda S, Heelan R and Zakowski MF: Solitary fibrous tumor. A cytologic-histologic study with clinical, radiologic, and immunohistochemical correlations. Cancer 81: 116-121, 1997.

12. Civin CI, Strauss LC, Brovall C, Fackler MJ, Schwartz JF and Shaper JH: Antigenic analysis of hematopoiesis. III. A hematopoietic progenitor cell surface antigen defined by a monoclonal antibody raised against KG-1a cells J Immunol 133: 157-165, 1984.

13. de Perrot M, Kurt AM, Robert JH, Borisch B and Spiliopoulos A: Clinical behavior of solitary fibrous tumors of the pleura. Ann Thorac Surg 67: 1456-1459, 1999.

14. Yokoi T, Tsuzuki T, Yatabe Y, Suzuki M, Kurumaya H, Koshikawa T, Kuhara H, Kuroda M, Nakamura N, Nakatani Y and Kakudo K: Solitary fibrous tumour: Significance of p53 and CD34 immunoreactivity in its malignant transformation. Histopathology 32: 423-432, 1998.

15. de Oliveira DH, Albuquerque AF, de Araújo Barreto MD, Nonaka CF, da Silva JS, Germano AR and Queiroz LM: Large solitary fibrous tumor of the oral cavity - report of a case. Pathol Res Pract 210: 1064-1067, 2014.

16. Ferretti GR, Chiles C, Choplin RH and Coulomb M: Localized benign fibrous tumors of the pleura. AJR Am J Roentgenol 169: 683-686, 1997.

17. Lee SC, Tzao C, Ou SM, Hsu HH, Yu CP and Cheng YL: Solitary fibrous tumors of the pleura: Clinical, radiological, surgical and pathological evaluation. Eur J Surg Oncol 31: 84-87, 2005.

18. Magdeleinat P, Alifano M, Petino A, Le Rochais JP, Dulmet E, Galateau F, Icard P and Regnard JF: Solitary fibrous tumors of the pleura: Clinical characteristics, surgical treatment and outcome. Eur J Cardiothorac Surg 21: 1087-1093, 2002.

19. Altinok T, Topçu S, Tastepe AI, Yazici U and Cetin G: Localized fibrous tumors of the pleura: Clinical and surgical evaluation. Ann Thorac Surg 76: 892-895, 2003. 